\title{
Fundamental and Practical Aspects of Thermal Analysis and Calorimetry
}

DOI: $10.1134 / \mathrm{S} 0036024420130191$

The XVI International Conference on Thermal Analysis and Calorimetry in Russia (RTAC-2020) was scheduled to take place in Moscow on July 5-9, 2020. The main organizers of the event are the Moscow State University (Lomonosov MSU), The Institute of General and Inorganic Chemistry (IGIC RAS, Moscow) and The National University of Science and Technology MISIS (NUST MISIS). However, the meeting is postponed until 2021.

The conference aims to provide an international forum for the worldwide community to meet, present and discuss fundamental and practical aspects of Thermal Analysis and Calorimetry, with special focus on the discussion of modern scientific and technological advances and methodological developments in this area of science, as well as the selection of the most promising methods and instruments and new directions of research. One feature of RTAC-2020 is that a significant part of the meeting will deal with computational methods, including CALPHAD and ab initio calculations.

The Conference Sessions are presented below:

(1) Inorganic substances and materials including complex compounds and natural minerals:

(a) Calorimetric methods (dissolution calorimetry, direct reaction calorimetry, adiabatic calorimetry, heat capacity determination, thermal analysis);

(b) Phase equilibria and phase transformations;

(c) Computational methods including CALPHAD and ab initio calculations.

(2) Organic and bio substances and materials:

(a) Calorimetric methods (dissolution calorimetry, direct reaction calorimetry, adiabatic calorimetry, heat capacity determination, thermal analysis);

(b) Phase equilibria and phase transformations;

(c) Computational methods.
(3) Equipment, methodology and standards of thermal analysis and calorimetry.

Most of the lecturers are leading scientists in different areas of calorimetry, thermal analysis and chemical thermodynamics. Representatives from Algeria, Australia, Azerbaijan, Belarus, China, France, Germany, Greece, India, Kazakhstan, Russia, Sweden, Tajikistan, Ukraine, and USA have submitted abstracts, and of course, intended to be present at the meeting this year. We had hoped that our Conference would be a good platform for discussion and to offer the opportunity to start new collaborations. The Organizing Committee has decided that the XVIth International Conference on Thermal Analysis and Calorimetry in Russia will be postponed and will now take place in the summer 2021 and will be linked to the RCCT Conference in Kazan. Nevertheless, the Book of Abstracts of RTAC-2020 has been published on the Conference site as originally planned, thanks to the generous financial support of Thermo-Calc Software AB.

The Organizing Committee expresses its gratitude to the Editorial Board of the Russian Journal of Physical Chemistry for its understanding and the assistance in publishing selected articles of RTAC-2020. The papers published as "Selected articles of RTAC-2020" are a good example of the broadness of the topics covered during the Conference as well as of its high scientific level.

On behalf of the Organizing Committee

Ivanov V.K., co-chair

Kalmykov S.N., co-chair

Gavrichev K.S., deputy chair

Uspenskaya I.A., deputy chair

Khvan A.V., deputy chair

Kovalenko N.A., scientific secretary 